\title{
Decellularizzazione d'organo: matrici fisiologiche per la generazione di organi in vitro
}

\section{Duccio Lombardi}

Dottorando in Farmacologia Preclinica e Clinica presso il Laboratorio Interdipartimentale di Nefrologia Cellulare e Molecolare, Centro DENOTHE, Univesità degli Studi di Firenze, Firenze

\begin{abstract}
ORGAN DECELLULARIZATION: A PHYSIOLOGICAL MATRIX FOR ORGAN REGENERATION IN VITRO
Abstract. Chronic kidney disorders are one of the most common current diseases, accounting for a large charge on National Health Services worldwide. Since these disorders usually progress in end-stage organ failure, patients will require replacement therapies such as transplantation. At the same time, the number of organ donors is inadequate and the risk of reject remains high. To overcome these problems, researchers are focusing on the development of new strategies for organ replacement. Among the solutions that are being tested, decellularization protocols of various organs are probably the most appealing ones. The biological rationale of these protocols is to obtain a biocompatible scaffold in which the patient's cells can be implanted with the purpose of restoring the organ's function, without creating a risk of rejection for the patient. This article will thus present the main characteristics of these protocols in the nephrologic area, in order to have an outlook of what might be the future of transplantation.
\end{abstract}

Key words: Decellularization, Organ replacement, In vitro organ creation, New therapeutic possibilities, Kidney

Conflict of interest: None.

Financial Support: None.

Ricevuto: 6 Luglio 2013; Accettato: 8 Luglio 2013

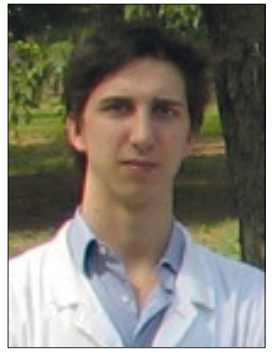

Duccio Lombardi

\section{Introduzione}

L'insufficienza renale cronica (o Chronic Kidney Disease, CKD) è una delle principali cause di mortalità e morbilità nei Paesi occidentali. Studi recenti riportano che la sua prevalenza, riferita pari al $13 \%$ circa nella popolazione adulta degli Stati Uniti e all'11\% in Europa, presenta un trend di crescita (1). Tale incremento è da ricercarsi non solo nel rapido aumento della diffusione di condizioni come l'obesità e il rigetto cronico del trapianto renale, ma soprattutto nella crescente incidenza di patologie croniche come il diabete mellito, patologie cardio-vascolari e infezione da HIV, che sono responsabili della maggior parte delle nefropatie (2).

L'insufficienza renale cronica è associata a un'elevata mortalità a causa del fatto che la maggior parte dei pazienti affetti da CKD evolve verso l'insufficienza renale terminale (o End Stage Renal Disease, ESRD), condizione non più suscettibile ad alcun tipo di terapia con intenti curativi. Di conseguenza, parallelamente all'incremento della prevalenza della CKD, è possibile riscontrare un contemporaneo aumento del numero di pazienti che progrediscono dalla malattia renale cronica verso l'insufficienza d'organo (3-5).

La naturale progressione della CKD verso la malattia renale terminale comporta la necessità di una terapia sostitutiva, rappresentata attualmente da dialisi extracorporea, dialisi peritoneale e trapianto di rene, $i$ cui costi di realizzazione e gestione superano consistentemente quelli applicati al trattamento di altre importanti patologie, come la malattia neoplastica (1). Parlando in numeri, nei soli Stati Uniti 100.000 individui sono in attesa di trapianto di rene, mentre circa un milione di pazienti soffre di ESRD e richiede, quindi, trattamenti dialitici. A ciò vanno poi aggiunte le 100.000 nuove diagnosi di CKD all'anno (6). Al fine di avere un quadro ancora più completo della gravità della situazione, va anche considerato che il tempo medio di attesa per un trapianto di rene negli USA è di 3 anni e che la mortalità in lista di attesa è pari al 5-10\%. Alle problematiche legate ai numeri di chi è in attesa di trapianto d'organo devono essere, inoltre, aggiunti i dati, sempre riferiti ai soli Stati Uniti, degli episodi di rigetto acuto $(20 \%$ dei trapiantati a 5 anni) e di perdita di funzionalità dell'organo 0 di morte del paziente (pari al 40\% a distanza di 10 anni) (7). 
Se, quindi, il quadro appare drammatico in un Paese con uno dei sistemi sanitari maggiormente all'avanguardia, si può ben immaginare come sia la situazione a livello mondiale, tanto da poter quasi azzardare il termine di pandemia.

Questi numeri non solo forniscono un'immagine dell'incidenza di CKD ed ESRD, ma, soprattutto, rendono l'idea di quanti sforzi debbano essere sostenuti dalle varie Sanità Nazionali per fare fronte alle spese necessarie per affrontare le diverse problematiche accennate. Se, quindi, come primo obiettivo vi è quello di assicurare una migliore qualità di vita al paziente, allo stesso tempo sorge la necessità di trovare rimedi che possano aiutare a contenere costi tanto elevati e con una forte incidenza sui bilanci sanitari statali. Dallo studio di soluzioni per tali due grandi soggetti, germoglia, quindi, il crescente impegno nello sviluppo di strategie che puntino a fornire organi funzionali e biocompatibili pronti per il trapianto. Tra queste soluzioni, quella a oggi maggiormente saggiata e promettente, in quanto capace di fornire impalcature biocompatibili su cui impiantare in seguito cellule dello stesso paziente, si basa sul concetto di decellularizzazione d'organo.

\section{Il concetto di decellularizzazione per la crea- zione di organi in vitro}

La necessità di fornire al paziente terapie che ne migliorino sempre più la qualità di vita e che, al contempo, permettano di ridurre le spese sanitarie sta fortemente influenzando la ricerca, alla quale sono richieste oggi soluzioni quanto più possibile permanenti e che risolvano sotto ogni punto di vista le varie problematiche del malfunzionamento d'organo. Da qui nasce il grande interesse per la creazione di organi in vitro.

La tecnica che suscita attualmente maggiori attrattive, come già accennato, è quella della decellularizzazione degli organi da donatori cadaverici. Tale processo permette di eliminare tutte le componenti cellulari dell'organo di interesse, mantenendo però intatta l'impalcatura dell'organo, costituita da proteine della matrice extracellulare (o ExtraCellular Matrix, ECM).

Ciò presenta una doppia finalità. In primis, quella di eliminare qualsiasi elemento che possa essere riconosciuto come non-self dal ricevente e che, di conseguenza, possa essere rigettato. In secondo luogo, questa tecnica fornisce una struttura a cui è possibile fornire cellule autologhe al fine di ricreare l'architettura e la funzionalità dell'organo, nella futura ottica della sostituzione biocompatibile.

In campo nefrologico, si è arrivati a parlare di decellularizzazione d'organo in seguito ad alcune recenti osservazioni. Esperimenti eseguiti in ratti anefrici, a cui erano impiantati sostituti costituiti da abbozzi renali, hanno dimostrato che tali reni primordiali si sviluppavano in organi funzionali, assicurando un prolungamento della vita del ratto (8). Altra osservazione di notevole rilievo è che tessuti di tratti urinari autologhi generati con matrici biocompatibili e cellule del paziente possono essere impiegati clinicamente come soluzione a varie patologie vescicali (9).

Queste e altre osservazioni hanno reso evidente come un punto chiave verso la creazione di organi permanentemente impiantabili passi dallo sviluppo di impalcature biocompatibili, o scaffold. Tali scaffold trovano come razionale biologico e finalità quella di fornire un'impalcatura che faciliti l'attecchimento e l'acquisizione di specifiche funzionalità da parte delle cellule che vi saranno sedimentate, permettendo al tempo stesso una piena integrazione dell'organo nel ricevente in assenza di una reazione immunitaria $(10,11)$.

Nel recente studio di Song et al. (11), è stato dimostrato come la matrice extracellulare, presente in ogni organo per sorreggerlo e dargli forma, possa fungere ottimamente e naturalmente da impalcatura per il successivo impianto di cellule. Ciò permetterebbe di generare organi funzionali e, allo stesso tempo, totalmente immunotollerabili, in quanto la ECM è costituita da proteine altamente conservate tra $\mathrm{i}$ mammiferi e non è, quindi, rigettabile.

Grazie all'impiego di detergenti, perfusi attraverso l'arteria renale a una pressione costante di $40 \mathrm{~mm} / \mathrm{Hg}$, gli $\mathrm{Au}-$ tori hanno dimostrato come sia possibile rimuovere tutte le componenti cellulari da reni cadaverici di ratto, maiale e uomo, senza influenzare funzionalità, proprietà biomeccaniche e caratteristiche delle proteine che compongono la matrice extracellulare. Sono state preservate, perciò, tutte le strutture che danno luogo ai sistemi vascolare, glomerulare e tubulare, anche se in assenza di cellule. Questo passaggio, preservando la struttura della ECM renale (la quale è fondamentale nei processi di filtrazione, secrezione e riassorbimento) ha, allo stesso tempo, permesso di eliminare tutte quelle componenti che potrebbero essere riconosciute come estranee dal sistema immunitario del ricevente. È inoltre da notare che, nello scaffold, il numero totale di strutture glomerulari rimaneva invariato, così come restavano invariati il diametro glomerulare, lo spazio di Bowman e la vascolatura dello stesso, pre-requisiti fondamentali affinché tali strutture possano essere correttamente ricolonizzate e tornare funzionali.

Una volta ottenuta l'impalcatura, gli Autori hanno scelto come fonti cellulari per la ripopolazione delle scaffold cellule endoteliali venose di cordone ombelicale umano (HUVECs), le quali sono state fornite via arteria renale, e cellule di rene neonatale di ratto (NKCs), fornite via uretere. Queste sono solo due tra molte possibili fonti cellulari e sicuramente molte altre soluzioni dovrebbero essere studiate e valutate per ottimizzare la resa dell'organo e per renderlo totalmente compatibile con il paziente. In questo caso, la scelta di cellule di ratto sarà spiegata in seguito, in quanto tali organi saranno trapiantati in ratti per eseguire test funzionali.

Inoltre, mentre nell'ingegneria di altri organi come muscoli e trachea la ripopolazione delle scaffold può avvenire per semplice sedimentazione cellulare o iniezione intraparenchimale, in un organo così complesso come il rene si è reso necessario, nonché logico, sfruttare la preesistente vascolatura e il compartimento urinario per fornire tali cellule $(12,13)$. Successivamente alla sedimentazione e all'incubazione per 3-4 giorni in bioreattori volti a fornire nutrienti, ossigenazione e stimoli alle cellule, gli Autori hanno osservato come le HUVECs ripopolassero le strutture vascolari dell'intero scaffold, partendo dalle arterie segmentali fino a giungere 
ai capillari glomerulari. Le NKCs epiteliali, invece, davano luogo in misura del $10 \%$ a cellule esprimenti podocina, ossia podociti, nel $70 \%$ dei casi a cellule positive per la pompa $\mathrm{Na}^{+} / \mathrm{K}^{+}$, tipica del tubulo prossimale, e nel $25 \%$ a cellule E-caderina positive, specifiche del tubulo contorto distale. Cosa maggiormente interessante è che tutte queste cellule avevano attecchito negli appropriati compartimenti dell'impalcatura e con la corretta polarità (vedi, per esempio, la distribuzione basolaterale della pompa $\mathrm{Na}^{+} / \mathrm{K}^{+}$). Sorge, quindi, spontaneo l'interrogativo: quali fattori determinano il corretto posizionamento delle cellule? Cosa spinge le cellule a differenziare nel fenotipo associato a quella data struttura? Queste tematiche saranno sicuramente oggetto di futuri studi, in quanto di fondamentale importanza per la comprensione dei fini meccanismi che permettono la creazione di tali sostituti d'organo.

Questi "reni rigenerati" sono stati, in seguito, testati sia in vitro che in vivo.

In vitro è stata valutata la capacità di ripulire un liquido di perfusione da metaboliti, di riassorbire elettroliti e glucosio e di generare urina concentrata. È stato, così, possibile osservare che la ritenzione di albumina era parzialmente ripristinata (46.9\% in più) rispetto al solo scaffold acellulare. Ciò indica una ripresa nella capacità filtrante glomerulare, la quale è notoriamente associata ai podociti. Analogamente, l'incremento nel riassorbimento del glucosio $(47.38 \%)$ denota la ripopolazione del tubulo prossimale da parte delle NKCs, che, differenziando, acquisiscono l'espressione di trasportatori funzionali di membrana tipici delle cellule epiteliali del tubulo prossimale stesso. Infine, l'aumento del $50 \%$ nel riassorbimento di elettroliti rispetto all'organo decellularizzato dimostra nuovamente che si ha il corretto attecchimento di cellule epiteliali che differenziano sia in unità funzionali del tubulo prossimale che dell'ansa di Henle che del tubulo distale.

I reni ri-cellularizzati sono stati successivamente impiantati in ratti sottoposti a nefrectomia unilaterale sinistra, con la finalità di eseguire analisi in vivo. Ciò ha permesso di dimostrare non solo che l'organo era ben perfuso, indicando una corretta ripopolazione di tutte le strutture vascolari, ma che produceva, inoltre, urina sin da poco dopo l'impianto. L'ultrafiltrato generato da tale organo presentava basse concentrazioni di creatinina e urea, ma, allo stesso tempo, miglioravano glicosuria e albuminuria rispetto all'organo non ri-cellularizzato, indicando il ripristino parziale delle principali unità filtranti renali: i podociti. La conclusione degli Autori è stata, quindi, quella di trovarsi di fronte a una capacità primordiale di clearance del sangue da parte di questi reni ricreati in laboratorio (11).

\section{Conclusioni}

Tutte queste evidenze supportano, quindi, l'ipotesi che l'utilizzo di impalcature derivate da organi decellularizzati e perciò, costituite da sole proteine della ECM, sia potenzialmente un'ottima soluzione per la creazione di organi in vitro. L'obiettivo rimane, ovviamente, sempre quello di poter ricreare tali organi con cellule derivate dallo stesso paziente, così da abbattere totalmente il rischio di rigetto, ottimizzando, al tempo stesso, le funzioni escretorie che, comunque, tale abbozzo renale già in parte presenta. Ciò lo renderebbe sicuramente un ottimo candidato dal punto di vista della sperimentazione clinica.

Ancora più interessante è il fatto che tali organi sembrano presentare una funzionalità sin dai primi momenti postimpianto, evento legato molto presumibilmente al fatto che le strutture della ECM glomerulare sono state sin da subito colonizzate dai podociti, così come le strutture tubulari sono state ripopolate da cellule epiteliali tubulari con corretta polarità. Ciò sottolinea l'importanza della ECM nell'indirizzare correttamente le diverse componenti cellulari per la rigenerazione tissutale.

Se, quindi, grandi sono i problemi sanitari connessi a patologie renali di natura cronica, sia per il paziente che da un punto di vista di spesa sanitaria, altrettanto grande interesse e ottime prospettive scaturiscono dagli studi bioingegneristici di decellularizzazione d'organo, al fine di creare organi sostituti, biocompatibili e, in futuro, immediatamente pronti all'impianto.

\section{Riassunto}

I disordini nefrologici cronici sono, a oggi, una tra le patologie a maggiore diffusione globale, la cui ricaduta economica ha profondi effetti sui Sistemi Sanitari Nazionali di tutto il mondo. Tali patologie di natura cronica generalmente progrediscono sino all'insufficienza d'organo, rendendo necessarie per il paziente terapie sostitutive come il trapianto d'organo. Allo stesso tempo, però, la richiesta di organi per il trapianto supera ampiamente la disponibilità degli stessi, motivo per cui la ricerca si sta sempre più focalizzando sullo sviluppo di nuove soluzioni che possano risolvere tale problematica. Una tra le soluzioni che riscuote, a oggi, maggiore successo è quella basata su protocolli che prevedono la decellularizzazione d'organo. La finalità di tali protocolli è quella di fornire impalcature biocompatibili su cui impiantare cellule dello stesso paziente, così da poter dare nuova e completa funzionalità all'organo e, allo stesso tempo, eliminare il rischio di rigetto da parte del ricevente. In questo articolo saranno, perciò, presentate, in campo nefrologico, le principali caratteristiche della decellularizzazione d'organo, così da poter avere una prospettiva di quello che potrebbe potenzialmente essere il futuro della medicina del trapianto.

Parole chiave: Decellularizzazione, Trapianto d'organo, Creazione di organi in vitro, Nuove possibilità terapeutiche, Rene

Dichiarazione di conflitto di interessi: L'Autore dichiara di non avere conflitto di interessi.

Contributi economici agli Autori: L'Autore dichiara di non aver ricevuto sponsorizzazioni economiche per la preparazione dell'articolo. 
Indirizzo degli Autori:

Dr. Duccio Lombardi

Centro per la Ricerca Trasferimento e Alta Formazione Studi a livello molecolare e clinico di malattie croniche, infiammatorie, degenerative e neoplastiche per lo sviluppo di nuove terapie (MCIDNENT)

Università degli Studi di Firenze

V.le G. Pieraccini 6

50139 Firenze

lombarduccio@alice.it

\section{Bibliografia}

1. Szczech LA, Lazar IL. Projecting the United States ESRD population: issues regarding treatment of patients with ESRD. Kidney Int Suppl 2004; 90: S3-7.

2. Macconi D, Abbate M, Morigi M, et al. Permselective dysfunction of podocyte-podocyte contact upon angiotensin II unravels the molecular target for renoprotective intervention. Am J Pathol 2006; 168: 1073-85.

3. Fox CS, Larson MG, Leip EP, Culleton B, Wilson PW, Levy D. Predictors of new-onset kidney disease in a community-based population. JAMA 2004; 291: 844-50.

4. United States Renal Data System. Annual data report: incidence and prevalence of ESRD. Am J Kidney Dis 2003; 42
(Suppl. 5): S37-173

5. Obrador GT, Pereira BJ. Epidemiology of Chronic Kidney Disease and screening recommendations. 2010; reperibile on-line sul sito www.uptodate.com.

6. Centers for Disease Control and Prevention. National chronic kidney disease fact sheet: general information and national estimates on chronic kidney disease in the United States, 2010.

7. Kawai T, Cosimi AB, Spitzer TR, et al. HLA-mismatched renal transplantation without maintenance immunosuppression. $\mathrm{N}$ Engl J Med 2008; 358: 353-61.

8. Rogers SA, Hammerman MR. Prolongation of life in anephric rats following de novo renal organogenesis. Organogenesis 2004; 1 (1): 22-5.

9. Atala A, Bauer SB, Soker S, Yoo JJ, Retik AB. Tissue-engineered autologous bladders for patients needing cystoplasty. Lancet 2006; 367 (9518): 1241-6.

10. Lombardi D. Generazione di strutture tissutali tridimensionali in vitro mediante l'uso di cellule staminali. TN\&D, 2012; 24 (2): 71-5.

11. Song JJ, Guyette JP, Gilpin SE, Gonzalez G, Vacanti JP, Ott HC. Regeneration and experimental orthotopic transplantation of a bioengineered kidney. Nat Med 2013; 19 (5): 646-51.

12. Quint C, Kondo Y, Manson RJ, Lawson JH, Dardik A, Niklason LE. Decellularized tissue-engineered blood vessel as an arterial conduit. Proc Natl Acad Sci U S A 2011; 108 (22): 9214-9.

13. Elliott MJ, De Coppi P, Speggiorin S, et al. Stem-cell-based, tissue engineered tracheal replacement in a child: a 2-year followup study. Lancet 2012; 380 (9846): 994-1000. 